\title{
A Case of Giant Cell Tumour of Bone in a Dog
}

\author{
Margaux Blondel ${ }^{1}$ Lucile Gros ${ }^{2}$ Marie-Odile Semi \\ André Autefage ${ }^{1 \odot}$ \\ ${ }^{1}$ Division of Small Animal Surgery, Department of Clinical Sciences, \\ Ecole Nationale Vétérinaire de Toulouse, University of Toulouse, \\ Toulouse, France \\ 2 Division of Diagnostic Imaging, Department of Clinical Sciences, \\ Ecole Nationale Vétérinaire de Toulouse, University of Toulouse, \\ Toulouse, France \\ ${ }^{3}$ Division of Pathology, Department of Biological and Fundamental \\ Sciences, University of Toulouse, Ecole Nationale Vétérinaire de \\ Toulouse, Toulouse, France
}

\begin{abstract}
Address for correspondence Sophie Palierne, DVM, MSc, PhD, Division of Small Animal Surgery, Department of Clinical Sciences, Ecole Nationale Vétérinaire de Toulouse, University of Toulouse, 23 Chemin des Capelles, BP 87614, 31076 Toulouse Cedex 3, France (e-mail: s.palierne@envt.fr).
\end{abstract}

VCOT Open 2019;2:e64-e69.

\begin{abstract}
Keywords

- giant cell

- bone tumours

- dogs

- curettage

- PMMA bone cement filling

Case Description A 9-year-old neutered female mixed-breed dog was presented for the assessment of right pelvic limb lameness of 1-week duration. The lameness had progressed to non-weight bearing the day before presentation.

Diagnostic Findings Radiographic examination of the right stifle joint revealed a large purely lytic lesion affecting the proximal metaphysis and epiphysis of the right tibia associated with a pathological fracture of the tibial plateau. These findings, combined with histological evaluation of a bone biopsy performed under ultrasoundguidance, were suggestive of a giant cell tumour of bone.

Treatment and Outcome A limb-sparing procedure was attempted as first-line treatment. Curettage of the tumour was followed by coblation, and the defect was filled with polymethyl methacrylate bone cement. Physical and radiographic follow-up examinations 1, 2 and 3 months after surgery revealed improved functional outcome and minor progression of osteolysis. However, local recurrence was apparent 5 months after initial surgery and the limb was therefore amputated.

Clinical Relevance Giant cell tumour of bone is rare in dogs. In humans, the traditional surgical management is curettage. This treatment was attempted here for the first time in a dog and the short-term outcome was satisfactory. However, in this particular case, local control of the tumour was unsuccessful.
\end{abstract}

\section{Introduction}

Giant cell tumour of bone (GCTB) is a common neoplasm in human patients and represents 4 to $5 \%$ of all primary bone tumours, and approximately $21 \%$ of benign bone tumors. ${ }^{1-4}$ In veterinary patients, however, this disease is relatively rare. ${ }^{5,6}$ Numerous authors have reported GCTBs in cats but only a few cases have been described in dogs so far. ${ }^{7-12}$

Giant cell tumours of bone frequently originate in the epiphysis of long bones. ${ }^{1-4}$ In humans, the most common sites include the distal femur, proximal tibia, distal radius and sacrum. ${ }^{1,2,13}$ In cats, two cases of GCTB originating from the diaphysis were diagnosed. ${ }^{8,10}$ In dogs, tumours affecting the accessory carpal bone ${ }^{7}$ and the distal humerus ${ }^{11}$ have been described.

Surgery is the treatment of choice in human patients and the tumour is commonly excised by curettage. ${ }^{1-3,13}$ To the authors' knowledge, this type of treatment has never been attempted in a dog. This is also the first report describing a GCTB of the proximal tibia in a dog.
License terms

Stuttgart · New York received
July 15,2019

accepted after revision

October 15, 2019
DOI https://doi.org/

10.1055/s-0039-3400513. ISSN $2625-2325$.

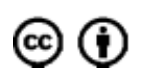




\section{Case Report}

A 9-year-old, $25 \mathrm{~kg}$ neutered female mixed-breed dog was presented for the assessment of an intermittent weightbearing right pelvic limb lameness of 1 -week duration, which had progressed to persistent non-weight-bearing lameness the day prior to presentation. No trauma was reported or change in general condition. As the dog had been adopted 1 year earlier, its complete medical history was not available.

General physical examination was within normal limits except for excess body weight (body condition score: 7/9). The initial orthopaedic examination revealed evident lameness combined with multiple rotational deformities of all four limbs. Severe amyotrophy of the right pelvic limb was detected, and a firm but subtle non-painful swelling was noted on the medial aspect of the right stifle joint. Signs of discomfort with bilateral hip extension were also apparent.

Radiography of the pelvis confirmed severe bilateral hip dysplasia associated with moderate osteoarthritis. Radiographs of the right tibia showed a large purely lytic lesion located in the caudal part of the proximal tibial epiphysis and metaphysis (-Fig. 1A, B). The lesion bulged beyond the confines of the caudal, lateral and medial cortices with eccentric erosion. Only a thin shell of subperiosteal bone outlined the outer surface of the defect caudally. The cranial margin of the lesion was round, smooth and regular, whereas the other borders were well-delimited but irregular. Proximally, the lesion extended to the proximal tibial subchondral bone, and a medial tibial plateau fracture was suspected.

Ultrasound-guided core needle bone biopsies (Tru-cut; MeritMedical, South Jordan, Utah, United States) were performed under sedation. Histopathological analysis of the samples did not reveal any bone structure, but showed a proliferation of densely packed, round to spindle-shaped cells (-Fig. 1C). Mild atypia was noted (anisocytosis, aniso- karyosis). Numerous giant cells with varying numbers of nuclei were dispersed throughout the section and were reminiscent of osteoclasts. Based on these radiographic and histopathological findings, a GCTB was diagnosed.

Routine haematology and biochemistry were unremarkable, and serum phosphate and ionized calcium levels were within normal values. Computed-tomography (CT) (Brightspeed Elite 16; General Electric Healthcare, Chicago, Illinois, United States) of both the thorax and the right stifle joint was performed, to exclude distant metastasis within the lungs and to better assess the tibial plateau. Computed tomography of the thorax was unremarkable. Sagittal and frontal CT images of the stifle ( - Fig. 2A, B) showed a collapse of the medial tibial plateau and confirmed the presence of an articular fracture. Curettage of the lesion, followed by filling the defect to treat the articular fracture and tibial plateau collapse, was attempted as first-line treatment.

A craniomedial approach to the proximal tibia was adopted, and the lesion was evaluated after dissecting its borders. A vast osteolytic region measuring $\sim 6 \mathrm{~cm}$ was identified. This region occupied both the epiphysis and metaphysis of the tibia and had a soft consistency that felt like 'egg shell crackling'.

Curettage of the tumour was achieved with a bone curette and aspiration. A gelatinous material was extracted and a fibrous membrane delimiting the tumour was visualized. The membrane was excised, and coblation (EVac Plasma Wand 70 Xtra, ArthroCare ENT; Smith \& Nephew, London, United Kingdom) was used to destroy the cells located on the borders of the lesion and to eliminate any remaining tumour tissues ( - Fig. 3A). Stay sutures were preplaced on the collapsed medial part of the tibial plateau to elevate the joint surface, and polymethyl methacrylate (PMMA) bone cement was used to fill the defect (-Fig. 3B). After copious lavage with sterile $0.9 \%$ saline solution, the surgical wound was routinely closed.
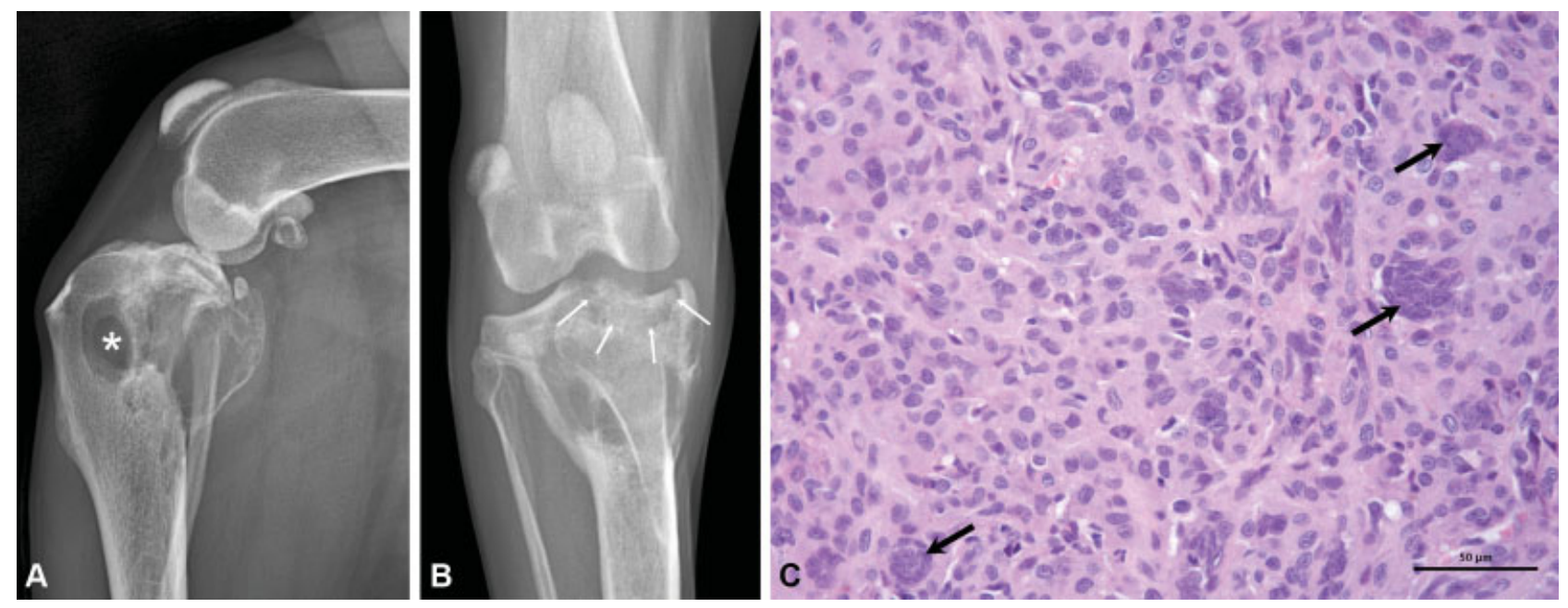

Fig. 1 Results of diagnostic tests done on admission: (A) Mediolateral and (B) craniocaudal radiographic projections of the right stifle joint. A large eccentric purely lytic lesion occupied the caudal part of the proximal epiphysis and metaphysis of the tibia. Caudal, lateral and medial cortices appeared blown and tapered, whereas the cranial margin of the lytic lesion was smooth and regular $\left({ }^{*}\right)$. No bone sclerosis was noticed. Proximally, the lesion extended to the tibial plateau subchondral bone, and a pathologic fracture of the tibial plateau was suspected (white arrows). (C) Histopathological analysis of a bone biopsy (haematoxylin and eosin, obj. $x 400$ ) revealed a proliferation of densely packed, round to spindle-shaped cells with mild cellular atypia. Dispersed throughout the section were numerous giant cells with varying numbers of nuclei (black arrows). 


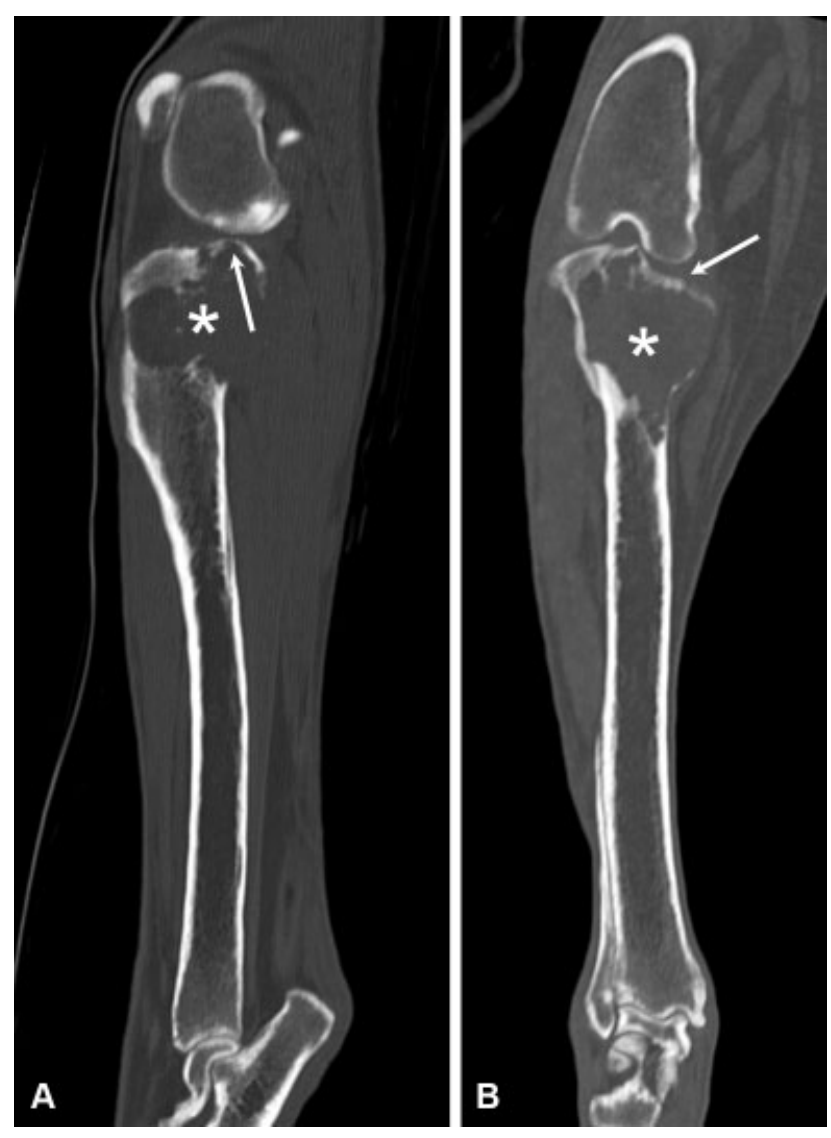

Fig. 2 Sagittal (A) and frontal (B) reformatted computed tomography images of the right stifle joint showed a wide eccentric lytic lesion of the tibia that extended proximally to the subchondral bone $\left({ }^{*}\right)$. Both images confirmed the medial tibial plateau collapse, without tumoural tissue invasion of the joint (white arrows). On the sagittal view, cranial to the area indicated by the arrow, a lytic line entering the joint space suggests a pathologic fracture of the tibial plateau.
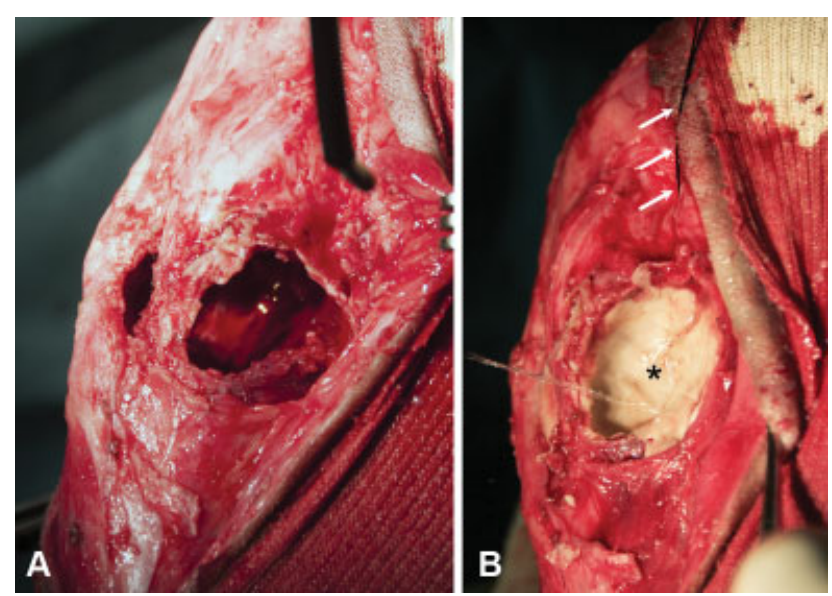

Fig. 3 Intraoperative images from the right proximal tibia. (A) Use of a Coblation wand (white arrow) to destroy any tumour tissues remaining on the margins of the lesion after curettage and excision of the membrane (B) Tibial plateau elevation with stay sutures (white arrows) and placement of polymethyl methacrylate bone cement within the defect (*).

Postoperative radiographs confirmed the restoration of joint congruity and correct filling of the defect with PMMA (-Fig. 4A, B). Persistent non-weight-bearing lameness was observed at discharge, 1 day after surgery. Oral firocoxib (Previcox; Merial, Lyon, France) (5 mg/kg orally once daily) was prescribed for 3 weeks, combined with absolute rest.

One month later, the owner reported a progressive improvement in functional recovery. Slight weight-bearing lameness of the right pelvic limb was still apparent on re-examination. A firm swelling on the medial side of the right knee was palpable, but the dog did not exhibit any pain during palpation of the proximal tibia, or manipulation of the knee. Radiographs showed a minor progression of osteolysis around the PMMA bone cement, and a subtle collapse of the tibial plateau was suspected medially.

Five months after surgery, the dog was reassessed for sudden non-weight-bearing right pelvic limb lameness. Radiographs of the right stifle joint showed evident signs of tumour recurrence as well as a tibial plateau fracture ( $\mathbf{F i g}$. 4C, D). It was finally decided to amputate the affected limb.

Sagittal osteotomy of the amputated tibia permitted an intralesional evaluation of the tumour. The PMMA bone cement was still in place, without any intra-articular extravasation, and was encircled by a gelatinous tumour tissue similar to that extracted during the initial surgery (-Fig. 5A). The tibial plateau fracture was also visible, and cartilaginous abrasions were detected on both femoral condyles ( - Fig. 5B). Histopathological analysis confirmed GCTB recurrence (-Fig. $\mathbf{5 C}$ ).

No lameness was apparent during the re-examination 4 months after amputation. Two months later, the dog was reassessed for sudden left pelvic limb lameness and for increasing difficulties in standing up and moving around. Except for the persistent excessive body weight, the general physical examination was unremarkable. The orthopaedic examination clearly revealed left pelvic limb lameness and pain was elicited upon manipulation of the left hip. Pelvic radiographs showed a progression of hip osteoarthritis. The owner declined radiography of the thorax. Routine serum biochemistry was unremarkable. Oral firocoxib (Previcox; Merial, Lyon, France) ( $5 \mathrm{mg} / \mathrm{kg}$ once daily) was administered once daily for 2 weeks, and weight loss and physiotherapy were strongly recommended. At the last follow-up, 13 months after amputation, the dog had lost weight and showed evident gait improvement. Three months later, the dog died for unknown reasons.

\section{Discussion}

Giant cell tumours of bone are rare in dogs. ${ }^{5,6}$ Clinical signs are non-specific, and include lameness and pain when pressure is applied to the affected region. Standard radiographs may be suggestive of the disease.

In humans, the basic radiographic image is that of pure osteolysis, affecting both the epiphyseal and metaphyseal regions of long bones. ${ }^{13}$ Spontaneous intrusion of the tumour into the adjacent joint remains exceptional. The tumour is often initially eccentric and periosteal reactions are rare. ${ }^{2,10}$ All cases of GCTBs reported in small animals seem to have these same characteristics. The radiographic images should not be mistaken for those of osteosarcomas, which remain the most common primary bone tumour in the dog. ${ }^{5}$ Their differentiation may be facilitated by the epiphyseal localization of 

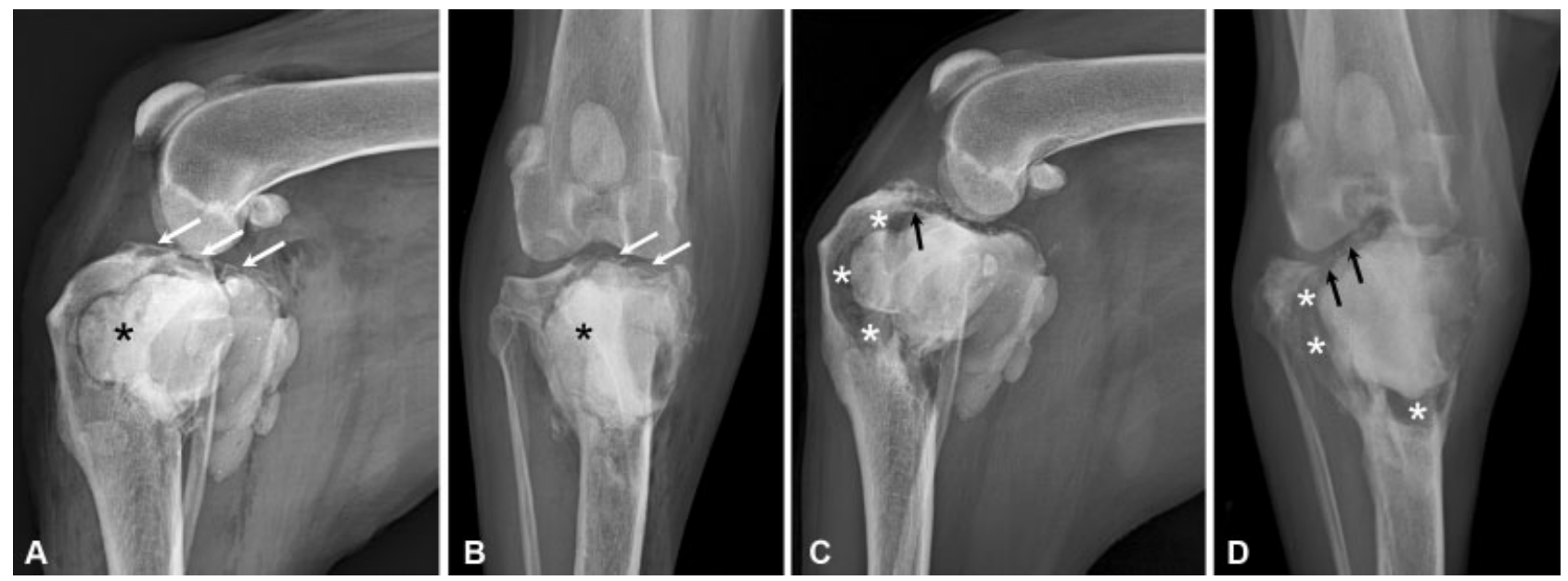

Fig. 4 Radiographic follow-up: (A) Mediolateral and (B) craniocaudal radiographic projections of the right stifle joint immediately after surgery. Polymethyl methacrylate (PMMA) bone cement $\left({ }^{*}\right)$ fills the complete lytic lesion. The medial tibial plateau collapse has been corrected (white arrows). No PMMA intra-articular penetration is visible. (C) Mediolateral and (D) craniocaudal radiographic projections of the right stifle joint at 5-month follow-up. Major progression of osteolysis around the PMMA bone cement is apparent $\left({ }^{*}\right)$. A lateral tibial plateau fracture could also be suspected (black arrows).
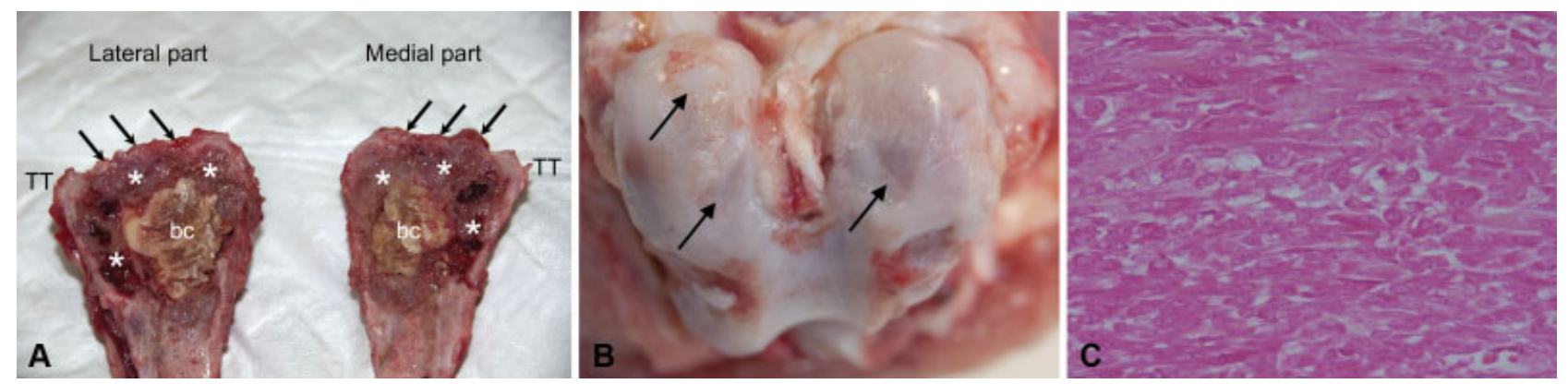

Fig. 5 Dissection of the right hindlimb after amputation and histopathological analysis of recurrent tumoural tissue. (A) Sagittal osteotomy of the right tibia. The polymethyl methacrylate (PMMA) bone cement (bc) is still in place, but a similar tissue to the one dissected during initial surgery was found surrounding the cement $\left({ }^{*}\right)$. The tissues also had the same gelatinous appearance. However, no extravasation of PMMA bone cement within the articular space was noticed (black arrows). TT=Tibial tuberosity. (B) Right femoral condyles, displaying cartilaginous abrasions (black arrows). These were most probably secondary to the joint incongruency caused by the medial and lateral tibial plateau collapse, and especially not to the cement given the absence of such material within the articular space. (C) Histopathological examination of the amputated specimen (haematoxylin and eosin, obj. x400), confirming giant cell tumour of bone recurrence. An intraosseous proliferation of fusiform cells with few atypia and few mitoses was visualized, similarly as that observed on the bone biopsies.

GCTBs and the absence of periosteal reactions. ${ }^{6,10,14}$ Codman's triangles are also very rarely seen. In our case, the preoperative radiographs showed an osteolytic lesion affecting both the metaphysis and the epiphysis of the proximal tibia, and a periosteal reaction was absent. A pathological fracture of the tibial plateau was also identified, which can occur in nine to $30 \%$ of human patients. ${ }^{15}$ Primary neoplasia was thought to be the most likely diagnosis, and more specifically an atypical osteosarcoma, given the extent of cortical destruction and lysis, as well as the localization of the lesion. However, because the lesion appeared cyst-like and benign with geometrical osteolysis on the radiographs, had a narrow transition area and was clearly-defined, a benign tumour could not be ruled out. The differential diagnosis also included solitary bone cyst, aneurysmal bone cyst, fibrous dysplasia of bone, plasma cell tumour and lymphoma. These diagnostic hypotheses were excluded following the histopathological analysis of bone biopsies prior to surgical treatment.
A classification of GCTBs, based on the lesions identified radiographically, has been established in human medicine. Three grades, correlated with local tumour aggressiveness and risk of local recurrence, have been described. ${ }^{1}$ In grade 1 , the cortical bone is intact, but it expands and becomes thinner (grade 2) before being destroyed completely (grade 3). Following this classification system, and given the blown aspect of the proximal tibial cortices as identified on the preoperative radiographs, a grade 3 would have been attributed in our case. The prognosis was, therefore, guarded.

Even if the radiograph is highly evocative, a biopsy is always required for definitive diagnosis. ${ }^{13}$ However, differentiating a GCTB from the giant cell-rich variant of osteosarcoma is, however, challenging because the histological features of both entities can overlap. ${ }^{16}$ These two tumour types cannot even be distinguished by staining cytological preparations for alkaline phosphatase activity. ${ }^{6}$ However, it should be possible to establish a diagnosis based on the 
absence of periosteal reaction on the radiographs and the very few atypia encountered during histological section examination. ${ }^{16}$ In humans, the immunohistochemical detection of a mutation-specific antibody directed against one particular mutant has also demonstrated high specificity and sensitivity for the diagnosis of GCTBs. ${ }^{17}$

If the cortices are not affected by osteolysis, and when sufficient intact subchondral bone is present, an additional imaging examination is not required. Otherwise, CT is advisable, because it offers better visualization of epiphyseal bone damage, particularly the condition of the subchondral bone, and because it is also the most sensitive means for detecting pulmonary metastasis. ${ }^{18}$ Indeed, even if GCTBs are considered benign, pulmonary metastasis occurs in 1 to $9 \%$ of human patients. ${ }^{1,19,20}$ Cases of dissemination to the lungs have also been described in small animals. ${ }^{8,12}$ In our case, CT permitted three-dimensional reconstruction of the stifle joint prior to surgery, which is particularly valuable when a limb-sparing procedure is selected.

Surgery is the standard treatment for GCTBs in human patients. ${ }^{1-3}$ However, because these tumours are subarticular, surgical resection can be invasive, and may lead to complications and impairment. Due to the benign character of these tumours, the best solution is an intralesional approach whenever possible. Such an approach has never been adopted in small animals. In our case, the dog's severe bilateral hip dysplasia, multiple rotational limb deformities, excessive body weight and the absence of lung metastasis prompted us to attempt to preserve the limb. This decision was further supported by the results of a study conducted on human patients with GCTBs and pathological fracture. In this cohort of patients, pain and functional outcome were greater after curettage than after resection. ${ }^{15}$

Curettage is in fact the traditional procedure undertaken in humans. ${ }^{1-3,13}$ It must be done meticulously as it determines the risk of recurrence. To ensure adequate curettage, the tumour is approached by making a large cortical window to allow the passage of curettes of appropriate size and type. ${ }^{13}$ Such a window was not necessary in our case, as the cortices on the craniomedial, caudomedial and caudolateral margins of the tumour were virtually absent.

The reported risk of local recurrence after curettage alone can be as high as $50 \%$ in human medicine. ${ }^{1,3,4}$ The mechanical effect of high-speed burring may thus help enlarge the margins of GCTB resection. ${ }^{21}$ Local use of adjuvants to enhance tumour destruction, such as liquid nitrogen, acrylic cement, phenol, argon beam, hydrogen peroxide, locally delivered chemotherapy or radiation therapy, has also been described in human medicine. ${ }^{1-3,13}$ No superior effect has been demonstrated so far, and malignant transformation has even been described after treatment with denosumab in human patients. ${ }^{22}$

In our case, although as aggressive curettage as possible was achieved, this approach could not be performed proximally, because of the possibility of causing further damage to the tibial plateau. High-speed burring was also not an option given the 'egg shell crackling' consistency of the tumour and the thinness of canine cortices. The risk of leaving tumour cells was high. We, therefore, chose to use coblation to destroy these remaining cells, as reported in the literature. ${ }^{23}$ Coblation is a low-temperature bipolar technique producing a plasma field at the tip of the electrode. The high-energy ionized plasma breaks the intramolecular bonds causing cavitation inside the tissues and subsequent decompression. To eliminate any remaining tumour tissue, the coblation probe was placed in contact with the entire wall of the tumour after curettage.

Reconstruction was necessary because of the joint surface fracture. Curettage of the tumour also created a consequent defect in the subperiosteal bone, further jeopardizing the integrity of the tibial plateau. Traditionally, bone graft or bone cement is used in humans. $1,2,13,24$ In the present case, and for several reasons, PMMA bone cement was chosen. The exothermic reaction, which occurs as the cement polymerizes, releases heat which may destroy any tumour cells remaining on the borders of the lesion after curettage and coblation, particularly under the tibial plateau where curettage was done with caution. 3,13,25 Subchondral replacement by PMMA bone cement also provided immediate restoration of joint congruity and therefore allowed early restoration of joint motion and weight bearing. Another advantage of using PMMA is related to the risk of recurrence. Local tumour recurrence was found to be minimal in human patients treated with PMMA cement as compared with bone grafting. ${ }^{13,24}$

The long-term prognosis regarding joint function after subchondral bone replacement by cement remains unknown. Curettage may have a deleterious effect on articular cartilage when it is performed adjacent to a joint. Moreover, articular damage caused by the exothermic reaction during cement polymerization has been described in humans, but remains unproven. $^{26,27}$ Even if osteoarthritis was radiographically evident in most of the patients treated with curettage and cementation in one study, it did not greatly affect their quality of life, suggesting a minor clinical impact. ${ }^{27}$ In the case described in this article, dissection of the right stifle joint after amputation revealed cartilaginous abrasions on both femoral condyles. However, as confirmed by the radiographs and necropsy, there was no PMMA bone cement extravasation occurred within the intra-articular space. These abrasions were, therefore, more likely to result from joint incongruency secondary to tibial plateau collapse and to expansile growth of the tumour at the time of recurrence, rather than to the cement itself.

Local recurrence remains the main risk after surgical management of GCTBs and most frequently occurs within 2 years after surgery. ${ }^{18,27}$ In our case, recurrence was somehow predictable. Indeed, as previously indicated, this dog had a grade 3 GCTB, which is associated in humans with the highest risk of local recurrence. ${ }^{1,13}$ Because such a classification does not exist in veterinary medicine, and due to the multiple orthopaedic anomalies, we suggested an attempt to excise the tumour surgically. Based on the improved functional outcome during the immediate postoperative period, and the progression of hip osteoarthritis revealed by the last follow-up examination, this treatment might have been successful in a dog with a lower grade GCTB.

Malignant transformation of GCTBs is rare, with a $4 \%$ incidence reported in human patients, and a 5-year survival 
rate of $87 \%{ }^{4}$ Even so, wide resection is recommended whenever recurrence occurs in humans ${ }^{13}$ and this is why the limb was finally amputated in the case reported here. Malignant transformation seemed unlikely given the relatively long-term follow-up ( $>13$ months), and was further confirmed by the histopathological analysis.

\section{Conclusion}

Giant cell tumour of bone is rarely reported in dogs. Even though radiographic findings, particularly the epiphyseal localization and purely osteolytic nature, can be evocative of this tumour, its differentiation from giant cell osteosarcoma based on a histopathological evaluation remains challenging. Given the biologically benign nature of these tumours, a limb-sparing procedure can be decided as first-line treatment with precise case selection. However, local recurrence remains the main risk after surgical treatment, even with adjuvant therapy, such as highlighted in this article.

\section{Authors' Contributions}

All authors contributed to conception of study, study design, acquisition of data data analysis and interpretation. Margaux Blondel, Sophie Palierne and André Autefage drafted, revised and approved the submitted manuscript. Lucile Gros, Marie-Odile Semin and Maxence Delverdier approved the submitted manuscript.

Funding

None.

\section{Conflict of Interest}

None of the authors of this paper has a financial or personal relationship with other people or organisations that could inappropriately influence or bias the content of the paper.

\section{References}

1 Sobti A, Agrawal P, Agarwala S, Agarwal M. Giant cell tumor of bone - an overview. Arch Bone Jt Surg 2016;4(01):2-9

2 Babinet A. Tumeurs à cellules géantes. In: Chotel F, Gouin F, eds. Tumeurs osseuses bénignes. Paris: Elsevier; 2005:201219

3 Skubitz KM. Giant cell tumor of bone: current treatment options. Curr Treat Options Oncol 2014;15(03):507-518

4 Palmerini E, Picci P, Reichardt P, Downey G. Malignancy in giant cell tumor of bone: a review of the literature. Technol Cancer Res Treat 2019;18:1533033819840000

5 Nielsen SW. Comparative pathology of bone tumors in animals, with particular emphasis on the dog. Recent Results Cancer Res 1976;54(54):3-16

6 Thompson KG, Dittmer KE. Tumors of bone. In: Meuten DJ, ed. Tumors in Domestic Animals. 5th edition. Iowa: John Wiley \& Sons, Inc.; 2017:356-424

7 Berg J, Gliatto JM, Wallace MK. Giant cell tumor of the accessory carpal bone in a dog. J Am Vet Med Assoc 1990;197(07):883-885

8 Ferreras MC, Fuertes M, Pérez V, et al. Giant cell tumour of bone in a cat with extraskeletal metastases: pathological and immuno- histochemical study. J Vet Med A Physiol Pathol Clin Med 2005;52 (05):225-229

9 Thornburg LP. Giant cell tumor of bone in a cat. Vet Pathol 1979;16 (02):255-257

10 Walsh BA, Rhodes WH. Giant cell tumour of bone in a cat. J Small Anim Pract 1995;36(07):325-329

11 LeCouteur R, Nimmo J, Price S, Pennock P. A case of giant cell tumor of bone (osteoclastoma) in a dog. J Am Anim Hosp Assoc 1978;14:356-362

12 Garman RH, Powell FR, Tompsett JW. Malignant giant cell tumor in a dog. J Am Vet Med Assoc 1977;171(06):546-548

13 Montgomery C, Couch C, Emory CL, Nicholas R. Giant cell tumor of bone: review of current literature, evaluation, and treatment options. J Knee Surg 2019;32(04):331-336

14 Thrall DE. Radiographic features of bone tumors and bone infections in dogs and cats. In: Thrall DE, ed. Textbook of Veterinary Diagnostic Radiology. 7th ed. St Louis: Elsevier; 2018:390-402

15 van der Heijden L, Dijkstra PD, Campanacci DA, Gibbons CL, van de Sande MA. Giant cell tumor with pathologic fracture: should we curette or resect? Clin Orthop Relat Res 2013;471(03):820-829

16 Chow LT. Fibular giant cell-rich osteosarcoma virtually indistinguishable radiographically and histopathologically from giant cell tumor-analysis of subtle differentiating features. APMIS 2015;123 (06):530-539

17 Schaefer IM, Hornick JL. Diagnostic immunohistochemistry for soft tissue and bone tumors: an update. Adv Anat Pathol 2018;25 (06):400-412

18 Armbrust LJ, Biller DS, Bamford A, Chun R, Garrett LD, Sanderson MW. Comparison of three-view thoracic radiography and computed tomography for detection of pulmonary nodules in dogs with neoplasia. J Am Vet Med Assoc 2012;240(09):1088-1094

19 Kremen TJ Jr, Bernthal NM, Eckardt MA, Eckardt JJ. Giant cell tumor of bone: are we stratifying results appropriately? Clin Orthop Relat Res 2012;470(03):677-683

20 Yang Y, Huang Z, Niu X, Xu H, Li Y, Liu W. Clinical characteristics and risk factors analysis of lung metastasis of benign giant cell tumor of bone. J Bone Oncol 2017;7:23-28

21 Algawahmed H, Turcotte R, Farrokhyar F, Ghert M. High-speed burring with and without the use of surgical adjuvants in the intralesional management of giant cell tumor of bone: a systematic review and meta-analysis. Sarcoma 2010;2010:1-5

22 Alaqaili SI, Abduljabbar AM, Altaho AJ, Khan AA, Alherabi JA. Malignant sarcomatous transformation of benign giant cell tumor of bone after treatment with denosumab therapy: a literature review of reported cases. Cureus 2018;10(12):e3792

23 Dabravolski D, Eßer J, Lahm A, Merk H. Surgical treatment of tumours and metastases of the spine by minimally invasive cavity-coblation method.J Orthop Surg (Hong Kong) 2017;25(01):2309499016684505

24 Zuo D, Zheng L, Sun W, Fu D, Hua Y, Cai Z. Contemporary adjuvant polymethyl methacrylate cementation optimally limits recurrence in primary giant cell tumor of bone patients compared to bone grafting: a systematic review and meta-analysis. World J Surg Oncol 2013;11:156

25 Nelson DA, Barker ME, Hamlin BH. Thermal effects of acrylic cementation at bone tumour sites. Int J Hyperthermia 1997;13 (03):287-306

26 Frassica FJ, Gorski JP, Pritchard DJ, Sim FH, Chao EY. A comparative analysis of subchondral replacement with polymethylmethacrylate or autogenous bone grafts in dogs. Clin Orthop Relat Res 1993;(293):378-390

27 van der Heijden L, van de Sande MA, Heineken AC, Fiocco M, Nelissen RG, Dijkstra PD. Mid-term outcome after curettage with polymethylmethacrylate for giant cell tumor around the knee: higher risk of radiographic osteoarthritis? J Bone Joint Surg Am 2013;95(21):e159 